\title{
Mutation Profile Assessed by Next-Generation Sequencing (NGS) of Circulating Tumor DNA (ctDNA) in Chinese Lung Adenocarcinoma Patients: Analysis of Real-World Data
}

\author{
Songchen Zhao $\mathbb{D}$, Xiaofeng Cong $\mathbb{D}$, and Ziling Liu \\ The First Hospital of Jilin University, China \\ Correspondence should be addressed to Ziling Liu; drzilingliu@163.com
}

Received 19 September 2020; Accepted 25 April 2021; Published 5 May 2021

Academic Editor: Marco De Martino

Copyright (c) 2021 Songchen Zhao et al. This is an open access article distributed under the Creative Commons Attribution License, which permits unrestricted use, distribution, and reproduction in any medium, provided the original work is properly cited.

\begin{abstract}
Background. Genomic testing gives guidance to the treatment options in lung adenocarcinoma patients, but some patients are unable to obtain tissue samples due to lesion location or intolerance. Cell-free circulating tumor DNA (ctDNA) tested in plasma or pleural effusion is an advanced access to solve the problem. Our study descriptively identified the genetic variations of advanced Chinese lung adenocarcinoma patients and analyzed the overall survival of patients with EGFR mutations. Methods. A total of 152 patients' plasma samples were included, and gene mutations were detected by NGS using an Illumina Miseq tabletop sequencer. Results. Frequencies of altered were EGFR 46.05\%, ALK 7.24\%, KRAS 6.58\%, PIK3CA 6.58\%, PTEN 2.63\%, HER2 $1.97 \%$, MET 1.97\%, BRAF 1.32\%, NF1 1.32\%, and ROS1 0.66\%. We identified 48 cases with double or triple driver gene mutations. Multiple mutations were more frequently observed in EGFR and PIK3CA genes. Patients harboring coexistent mutations with an EGFR mutation tended to have a shorter overall survival than those with exclusively EGFR mutations. Conclusion. EGFR, ALK, and KRAS were common driver gene in Chinese patients with stage IV lung adenocarcinoma. Multiple mutations were detected in the ctDNA samples and involve more EGFR and PIK3CA mutations. The existence of coexisting gene mutations may have adverse effects on the prognosis of patients with EGFR mutation. The unknown mutations discovered by NGS may provide new targets for gene targeting therapy, and ctDNA test by NGS is an effective method for making appropriate treatment choices.
\end{abstract}

\section{Introduction}

At present, lung cancer is still the leading cause of cancer incidence and mortality worldwide [1]. However, the mortality of lung cancer has dropped in the last decade which is related to the development of targeted therapies and other advanced therapies [2]. Targeted molecular therapy has improved the outcomes of patients with advanced nonsmall cell lung cancer (NSCLC) who harbor sensitive mutations $[3,4]$. Therefore, genomic testing is crucial to explore potential molecular targets for the treatment of lung cancer, thereby reducing the mortality of NSCLC. The National Comprehensive Cancer Network treatment guidelines advocate actionable mutation screening as standard of care, and genomic retesting is necessary at a time of tumor progression [5]. However, tissue samples are often difficult to obtain due to lesion location or intolerance, especially in relapsed and metastatic settings. Liquid biopsy is a noninvasive, clinically actionable, and reliable method to solve the problem. NGS uses ctDNA shed from tumors into the circulation as a substrate for mutation detection. Recent studies showed that plasma NGS testing demonstrates a marked increase of the detection of therapeutically targetable mutations $[6,7]$. Furthermore, the detection of ctDNA by NGS allows huge amounts of variants to be identified in each sample on a single platform. In our study, we identified the genomic mutation profile of ctDNA in real-world Chinese stage IV lung adenocarcinoma patients using the NGS panel. 
TABle 1: Demographic characteristic of patients.

\begin{tabular}{|c|c|c|c|c|c|c|c|c|c|c|c|c|}
\hline & \multicolumn{2}{|c|}{ EGFR } & \multirow[b]{2}{*}{$p$} & \multicolumn{2}{|c|}{ ALK } & \multirow[b]{2}{*}{$p$} & \multicolumn{2}{|c|}{ PIK3CA } & \multirow[b]{2}{*}{$p$} & \multicolumn{2}{|c|}{ KRAS } & \multirow[b]{2}{*}{$p$} \\
\hline & + & - & & + & - & & + & - & & + & - & \\
\hline \multicolumn{13}{|l|}{ Gender } \\
\hline Male & 34 & 46 & \multirow{2}{*}{0.354} & 6 & 74 & \multirow{2}{*}{0.895} & 6 & 74 & \multirow{2}{*}{0.877} & 6 & 74 & \multirow{2}{*}{0.877} \\
\hline Female & 36 & 36 & & 5 & 67 & & 4 & 68 & & 4 & 68 & \\
\hline \multicolumn{13}{|c|}{ Age (years) } \\
\hline$N<60$ & 35 & 35 & \multirow{2}{*}{0.174} & 6 & 61 & \multirow{2}{*}{0.681} & 5 & 62 & \multirow{2}{*}{0.952} & 3 & 64 & \multirow{2}{*}{0.550} \\
\hline$N \geq 60$ & 32 & 50 & & 5 & 80 & & 5 & 80 & & 7 & 78 & \\
\hline
\end{tabular}

\section{Methods}

2.1. Patients. A total of 152 patients who were pathologically diagnosed with stage IV lung adenocarcinoma were included in our study. These eligible patients received NGS assays in The First Hospital of Jilin University from January 2016 to December 2019. 14 genes (EGFR, ALK, KRAS, PIK3CA, PTEN, HER2, MET, BRAF, AKT1, NF1, ROS1, RET, NRAS, and MAP2K1) were detected for 94 patients, 139 genes were detected for 25 patients and 425 genes for 33 patients, and the choices were made by patients and their physicians.

2.2. Blood Samples and ctDNA Extraction. $5 \mathrm{ml}$ of whole blood was collected by ethylenediamine tetraacetic acid (EDTA) blood collection tubes then transported at ambient temperature to Nanjing Shihe Jiyin Biotech Inc. (Nanjing, China) no more than $72 \mathrm{~h}$. Blood was centrifuged at $1800 \times \mathrm{g}$ for 10 minutes at $4^{\circ} \mathrm{C}$ to remove blood cells. Then, the supernatant was centrifuged at $16000 \times \mathrm{g}$ for 10 minutes at $4^{\circ} \mathrm{C}$ to remove any remaining cells. Circulating tumor DNA was extracted from $2 \mathrm{ml}$ plasma, by digestion in $100 \mu \mathrm{l}$ proteinase $\mathrm{K}$ buffer for $10 \mathrm{~min}$ at $37^{\circ} \mathrm{C}$ followed by purification with the NucleoSpin Plasma XS kit with modified protocols. The purified ctDNA is quantified by a Picogreen fluorescence assay using the provided lambda DNA standards (Thermo Fisher Scientific, Waltham, MA, USA).

2.3. ctDNA Sequencing and Analysis. The 5'-biotinylated probe solution is provided as capture probes, and the baits target cancer-related genes. Hybridization, target amplifcation, barcode library preparation, and size selection were performed according to the manufacturer's protocols. After amplification, the samples are purified by AMPure XP beads, quantified by qPCR (Kapa Biosystems, Boston, USA), and sized on bioanalyzer 2100 (Agilent Technologies, China). Libraries are normalized to $2.5 \mathrm{nM}$ and pooled. Deep sequencing is performed on Illumina HiSeq 4000 using PE75 V1 Kit. Cluster generation and sequencing are performed according to the manufacturer's protocol.

Base calling was performed using bcl2fastq (Illumina, Inc. San Diego, California, USA) to generate sequence reads in FASTQ format (Illumina, Inc. San Diego, California, USA). Quality control was applied with Trimmomatic [8]. High-quality reads were mapped to Human Genome Build 19 (Hg19)/GRCh 37 reference sequence. Single nucleotide variants and short insertions/deletions were identified using
VarScan2 [9], and copy number variations (CNVs) were identified using ADTEx [10]. All the above experimental steps were carried out by Nanjing Shihe Jiyin Biotech Inc. (Nanjing, China).

2.4. Data Collection and Statistics. Demographic characteristics of the patients were collected from the medical records from The First Hospital of Jilin University. Patients that harbored EGFR mutations were treated with EGFR-TKIs for first-line treatment while the others with chemotherapy. Overall survival (OS) was defined as the time from the molecular analysis assessment date to the date of death or final follow-up (2021.01.09). Survival curves were estimated by the Kaplan-Meier method for the patients with EGFR mutations. The relationship between mutation status and patient characteristics was compared by using the chi-square test for qualitative variables or a nonparametric test for quantitative variables. All data were analyzed by SPSS version 25.0 (IBM Corporation, Armonk, NY, USA), $p<0.05$ was considered to be statistically significant.

\section{Results}

3.1. Patient Characteristics and Gene Mutation Patterns. 152 patients with stage IV lung adenocarcinoma were included in this study. The cohort included 80 male patients and 72 female patients; 86 were over 60 years old and 66 under 60 years old (Table 1). With regard to the 14 genes tested in all patients, 109 patients $(71.7 \%)$ harbored at least one genomic mutation. Frequency of altered was EGFR 46.05\%, ALK 7.24\%, KRAS 6.58\%, PIK3CA 6.58\%, PTEN 2.63\%, HER2 $1.97 \%$, MET $1.97 \%$, BRAF $1.32 \%$, NF1 $1.32 \%$, and ROS1 $0.66 \%$ (Figure 1). The EGFR mutation was more common in females, $<60$ years, but in our cohort, these differences were not statistically significant $(p>0.05)$ (Table 1$)$. There was no difference among EGFR (-) vs. exclusively EGFR mutated cases vs. EGFR + coexistent mutations with sex and age (Table 2).

3.2. Distribution of Common Gene Mutations. EGFR mutations were detected in 70 patents of the total 152 patients, including 34 who harbored double or triple EGFR gene mutations. The most common EGFR mutations were exon19 deletions (51.72\%, 15/29), followed by L858R (34.48\%, $10 / 29$ ) in patients who harbored single mutation (Table 3 ). 17 patients with tumor recurrence harbored T790M mutation, and we also found one untreated patient detected with 


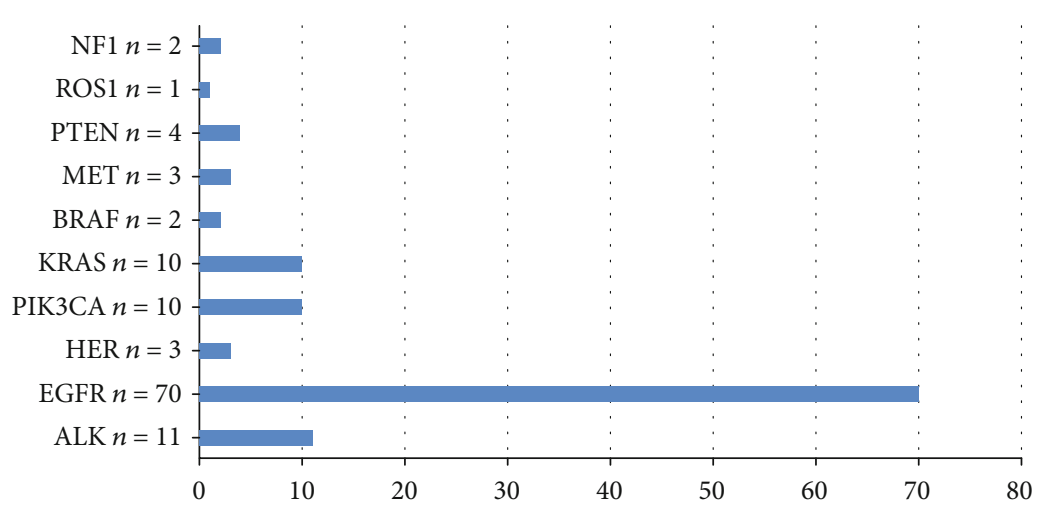

Figure 1: Mutation of common driver genes.

TABle 2: The relationship between EGFR mutation status and patients' characteristics.

\begin{tabular}{ccccc}
\hline & $\begin{array}{c}\text { EGFR } \\
(-)\end{array}$ & $\begin{array}{c}\text { Exclusively } \\
\text { EGFR mutations }\end{array}$ & $\begin{array}{c}\text { EGFR +coexistent } \\
\text { mutations }\end{array}$ & $p$ value \\
\hline Gender & & & & 0.252 \\
Male & 36 & 32 & 5 & \\
Female & 46 & 26 & 8 & \\
Age (years) & & & & 0.448 \\
$N<60$ & 32 & 28 & 4 & \\
$N \geq 60$ & 50 & 30 & 8 & \\
\hline
\end{tabular}

TABLE 3: Single mutation of EGFR gene.

\begin{tabular}{lcc}
\hline EGFR gene mutations & Number of patients & Percentage (\%) \\
\hline Exon-19 del & 15 & 51.72 \\
Exon-21 L858R & 10 & 34.48 \\
Exon-19 ins & 1 & 3.45 \\
Exon-20 ins & 1 & 3.45 \\
Exon-21 L861Q & 1 & 3.45 \\
Gene amplification & 1 & 3.45 \\
Total analyzed & 29 & 100.00 \\
\hline
\end{tabular}

In our cohort, exon-19 (62.16\%) was the most common EGFR gene mutation, followed by exon-21 L858R mutation (27.02\%).

T790M mutation. In addition, insert mutation of exon-19, exon-20, L861Q mutation, and copy number amplification was detected in 1 case each. EML-4/ALK fusion (8/11) was the most common mutation in the ALK gene. The remaining of these is located in exon-19 or exon-20. PIK3CA mutations were detected in 10 patients, most of those located on E545K in exon-9(6/10), and the others in exon-20. KRAS mutations were identified in 10 patients, and all of them were on exon-2. G12C (5/10) was the most frequent mutation detected in the KRAS gene. PTEN mutations were detected in 6 patients, 1 of them was truncation mutation, and the others were point mutations. HER2 mutations: insertion mutation in exon-20 ( 2 cases) and germ line mutation ( 1 case) were identified in 3 cases. BRAF mutations were identified in 2 patients; all of them were V600E mutation. 2 cases of NF1 mutations and 1 case of ROS1 fusion mutation were also detected. The specific distribution was shown in Table 4.

3.3. Multiple and Unknown Gene Mutations Detected in ctDNA of Stage IV Lung Adenocarcinoma. Out of 109 patients with genetic variations, 43 patients were found to harbor multiple mutations (29 exclusively EGFR mutations and 14 coexisting mutations). Of the exclusively double or triple EGFR mutations, most were EGFR sensitive mutation (exon - 19 deletion/exon - 21 L858R) + T790M (11/34), followed by exon -19 deletion + gene copy number amplification (3/34). Coexistent mutations were detected in 14 patients: 4 EGFR+PIK3CA, 1 EGFR+PIK3CA + PTEN, 3 EGFR + PTEN, 1 EGFR+HER2, 1 EGFR+KRAS, 1 PIK3$\mathrm{CA}+\mathrm{HER} 2, \quad 1$ PIK3CA + KRAS, 1EGFR + NF1, and 1 EGFR + MET (Table 5); in this small cohort, EGFR remains the most common mutation gene (12/14) followed by PIK3CA (7/14). We also detected 13 patients with gene mutations whose functions were still unclear (Table 6).

3.4. Overall Outcome in Patients Harboring EGFR Mutations. Out of 70 patients harboring EGFR mutations, 58 were exclusively EGFR mutations ( 29 single EGFR mutations and 29 double or triple EGFR mutations), and 12 were coexistent mutations. All of these patients received TKI therapy as first line treatment. After the exclusion of 11 patients who were lost to follow-up, survival data were obtained in 59 patients. Patients harboring coexistent mutations with an EGFR mutation tended to experience worse prognosis than those with exclusively EGFR mutations ( $\mathrm{OS}=21.0$ vs. 16.0 months, $p=0.104$, Figure 2 ) although not statistically significant.

\section{Discussion}

We tested 152 Chinese stage IV lung adenocarcinoma liquid samples to analysis gene mutation patterns by NGS. High throughput, multiplex tests implementable for clinical are necessary to direct therapy choice for individual patients. Several studies have demonstrated that NGS is a capable method, which is quick, stable, and cost-effective [11-14]. Furthermore, NGS provided both the advantage of low input DNA concentration and the detection of low-frequency 
TABLE 4: Specific distribution of common gene mutations except for EGFR mutations.

\begin{tabular}{|c|c|c|c|}
\hline Gene & Mutation & Number & Percentage (\%) \\
\hline \multirow{4}{*}{ ALK } & EML4-ALK & 8 & 5.26 \\
\hline & $\mathrm{D} 1311 \mathrm{E}$ & 1 & 0.66 \\
\hline & $\mathrm{K} 1101 \mathrm{~N}$ & 1 & 0.66 \\
\hline & $\begin{array}{l}\mathrm{NT5C1B} / \mathrm{MIR} 4757 \text { break } \\
\text { and rearrangement }\end{array}$ & 1 & 0.66 \\
\hline \multirow{5}{*}{ PIK3CA } & E545K & 6 & 3.95 \\
\hline & H1047L & 1 & 0.66 \\
\hline & P449L & 1 & 0.66 \\
\hline & M1043I & 1 & 0.66 \\
\hline & Q546K & 1 & 0.66 \\
\hline \multirow{5}{*}{ KRAS } & G12C & 5 & 3.29 \\
\hline & G13D & 2 & 1.32 \\
\hline & G12A & 1 & 0.66 \\
\hline & G12D & 1 & 0.66 \\
\hline & G12V & 1 & 0.66 \\
\hline \multirow{6}{*}{ PTEN } & S59X & 1 & 0.66 \\
\hline & A126T & 1 & 0.66 \\
\hline & R130X truncation & 1 & 0.66 \\
\hline & V317 & 1 & 0.66 \\
\hline & A126T & 1 & 0.66 \\
\hline & L247 & 1 & 0.66 \\
\hline \multirow{2}{*}{ HER2 } & Exon-20 insertion & 2 & 1.32 \\
\hline & R143Q & 1 & 0.66 \\
\hline MET & Amplification & 3 & 1.97 \\
\hline \multirow{2}{*}{ NF1 } & $\mathrm{W} 2317 \mathrm{X}$ truncation $+\mathrm{Y} 489 \mathrm{C}$ & 1 & 0.66 \\
\hline & S436fs & 1 & 0.66 \\
\hline BRAF & V600E & 2 & 1.32 \\
\hline ROS-1 & ROS1-CD47 fusion & 1 & 0.66 \\
\hline Total & & 50 & 32.9 \\
\hline
\end{tabular}

variants [12]. Therapies matched to ctDNA mutations monitoring during treatments demonstrated appreciable therapeutic efficacy [15].

Previous studies showed that the most common EGFR mutations in NSCLC were exon-19 deletions and point mutation L858R in exon-21, which were referred to as sensitive mutations and benefited from RGFR-TKIs' therapy [16]. We conducted genomic test in 152 Chinese patients; in our cohort, the EGFR sensitive mutation rate was significantly higher than those declared in American patients with lung adenocarcinomas (46.05\% vs. $19.0 \%$ ) [17]. It shows that ethnic difference exists in the distribution of gene mutations. As EGFR sensitive mutations were more likely to occur in lung adenocarcinoma [16], our incidence was higher than previously reported Chinese NSCLC patients (46.05\% vs. 34.8\%) [18]. We detected a case of L681Q mutation on exon-21 which was considered very rare. Patients with L681Q mutations showed poor outcomes compared with those who har- bored sensitive mutations $[19,20]$. Inspiringly, several clinical trials have demonstrated that afatinib was active in patients' harbored L681Q mutation [21, 22]. T790M mutation was always acquired in lung adenocarcinoma patients after exposure to EGFR-TKIs' therapies, which related to resistance to the first or second generation of TKIs [23]. In our study, 21 patients with T790M mutations were identified including a case without TKI therapy history. EGFR gene mutations were observed commonly in female, $<60$ years, and adenocarcinoma patients with NSCLC in previous reports [18]. In our cohort, EGFR mutation was not significantly correlated with age and gender. Our small sample size and fixed pathologic type may result to the inconsistent. And the $p$ value is near 0.05 for gender difference, which may be due to the limited sample sizes. In our cohort, the mutation status of EGFR was not associated with age $(p>0.05)$ (Table 2). EGFR negative and EGFR + coexistent mutations appear to be more common in females, whereas exclusively EGFR-mutated cases were more likely to occur in males (Table 2), but the differences were not statistically significant.

The incidence of ALK gene fusion mutation was about $3.8 \%$ in the Asian lung adenocarcinoma population [24]. In our cohort, 9 of 152 patients with stage IV lung adenocarcinoma had positive ALK gene fusion mutations, with a mutation rate of $7.24 \%$, slightly higher than the reported level. Almost all the ALK gene fusion mutations detected were EML4-ALK fusion mutations (8/11). 2 cases of unknown point mutations (D1311E, K1101N) on the ALK gene were detected, whose function was still unclear in the development of tumor.

The incidence of KRAS gene mutation was $15 \%-25 \%$ in patients with nonsmall cell lung cancer [25]. In our cohort, 10 cases with KRAS gene mutation were detected in 152 patients with stage IV lung adenocarcinoma, with a mutation rate of $6.58 \%$, slightly lower than the reported level. Most KRAS gene mutations detected were on codon 12 of exon 2 $[26,27]$, which was associated with a poor prognosis and resistance to TKI therapies.

The incidence of PIK3CA gene mutation in nonsmall cell lung cancer is $2 \%-5 \%[18,28,29]$. In 152 patients with stage IV lung adenocarcinoma, 10 were detected with PIK3CA mutations (6.58\%). Most mutations occurred in exon 9 or $20(6 / 4)$, and 7 cases of PIK3CA mutations coexisted with other drive genes at the same time: 5 PiK3CA + EGFR, 1 PIK3CA + HER2, and 1 PIK3CA+ KRAS. In our cohort, PIK3CA mutations tended to accompany other driver genes such as EGFR and KRAS coexist and always occur on exon 9 or 20 , and these results showed consistency with a previous study [29].

In the past, it is wildly believed that lung cancer drive gene mutations were mutually exclusive [30-32]. With the development of gene detection technology, cases of driver gene mutations coexistence have been detected. In a study of 5125 Chinese NSCLC patients, 160 multiple genetic mutations were found including EGFR+PIK3CA, EGFR+ KRAS, KRAS+PIK3CA, EGFR+BRAF, PIK3CA + BRAF, and EGFR+KRAS +PIK3CA [18]. In our cohort of 14 patients with multiple gene mutations coexistence, 12 patients were detected with EGFR mutations, and the 
TABLE 5: Combinations of multiple gene coexistence mutations.

\begin{tabular}{|c|c|c|c|c|}
\hline Mutation 1 & Mutation 2 & Mutation 3 & Mutation 4 & Number \\
\hline EGFR 19 del & EGFR A750P & & & 1 \\
\hline EGFR 19 del & EGFR E922V & & & 1 \\
\hline EGFR 19 del & EGFR T790M & & & 6 \\
\hline EGFR 19 del & EGFR T790M & EGFR gene amplification & & 1 \\
\hline EGFR 19 del & EGFR T790M & EGFR C797S & & 1 \\
\hline EGFR 19 del & EGFR gene amplification & & & 3 \\
\hline EGFR 19 del & EGFR R689W & & & 1 \\
\hline EGFR 19 del & EGFR S752 & & & 1 \\
\hline EGFR 20ins & EGFR IGFBP3\&LOC7 confusion & EGFR gene amplification & & 1 \\
\hline EGFR 20ins & EGFR V774 & & & 1 \\
\hline EGFR 21L858R & EGFR T790M & & & 5 \\
\hline EGFR 21L858R & EGFR E790K & & & 1 \\
\hline EGFR 21L858R & EGFR T790M & EGFR L62R & & 1 \\
\hline EGFR 21L858R & EGFR T790M & EGFR T725M & EGFR gene amplification & 1 \\
\hline EGFR 21L885R & EGFR gene amplification & & & 2 \\
\hline EGFR 21L858R & EGFR L62R & & & 1 \\
\hline EGFR 21L858R & EGFR L833V & & & 1 \\
\hline EGFR 19 del & PIK3CA E542K & & & 1 \\
\hline EGFR 19 del & PIK3CA E545K & & & 1 \\
\hline EGFR 19 del & PIK3CA E545K & EGFR T790M & EGFR C797S & 1 \\
\hline EGFR 19 del & PIK3CA M1043 & PTEN S59X & EGFR T790M & 1 \\
\hline EGFR 21 L858R & PIK3CA E545K & & & 1 \\
\hline EGFR 19 del & PTEN A126T & & & 1 \\
\hline EGFR 19 del & PTEN L247fs & EGFR T790M & & 1 \\
\hline EGFR 19 del & HER2 gene amplification & EGFR gene amplification & & 1 \\
\hline EGFR 19 del & KRAS G13D & & & 1 \\
\hline HER2 p.771insAYVM & PIK3CA P449L & & & 1 \\
\hline KRAS G12C & PIK3C A H1047L & & & 1 \\
\hline EGFR 19 del & NF1 S436fs & & & 1 \\
\hline EGFR 19 del & HER2 R143Q & & & 1 \\
\hline EGFR 19 del & MET gene amplification & EGFR E922V & & 1 \\
\hline Total & & & & 43 \\
\hline
\end{tabular}

TABLE 6: 13 patients were detected with unknown mutations.

\begin{tabular}{|c|c|c|c|c|c|}
\hline Case & Mutation 1 & Mutation 2 & Mutation 3 & Mutation 4 & Mutation \\
\hline 1 & RNF43 fusion & & & & \\
\hline 2 & AKT2 P24S & GNA11 R114Q & RB1 I181V & BRIP1 M1V & \\
\hline 3 & MYC S154L & & & & \\
\hline 4 & AXL-IGR fusion & SP0P L149I & TP53 P98Lfs & & \\
\hline 5 & SMAD4 D424N & & & & \\
\hline 6 & DNMT3A H873R & & & & \\
\hline 7 & NRAS D47H & & & & \\
\hline 8 & SMO G177C & & & & \\
\hline 9 & APC I224M & & & & \\
\hline 10 & CBL E693V & FLT3 W196C & KDR N580D & NF2 A164V & \\
\hline 11 & GNAS R632C & IKBKE F224V & & & \\
\hline 12 & ATRX S1153L & EPHA3 fusion & & & \\
\hline 13 & PRKCI E559X truncation & TGFBR2 G399R & INPP4B Q811E & & \\
\hline
\end{tabular}




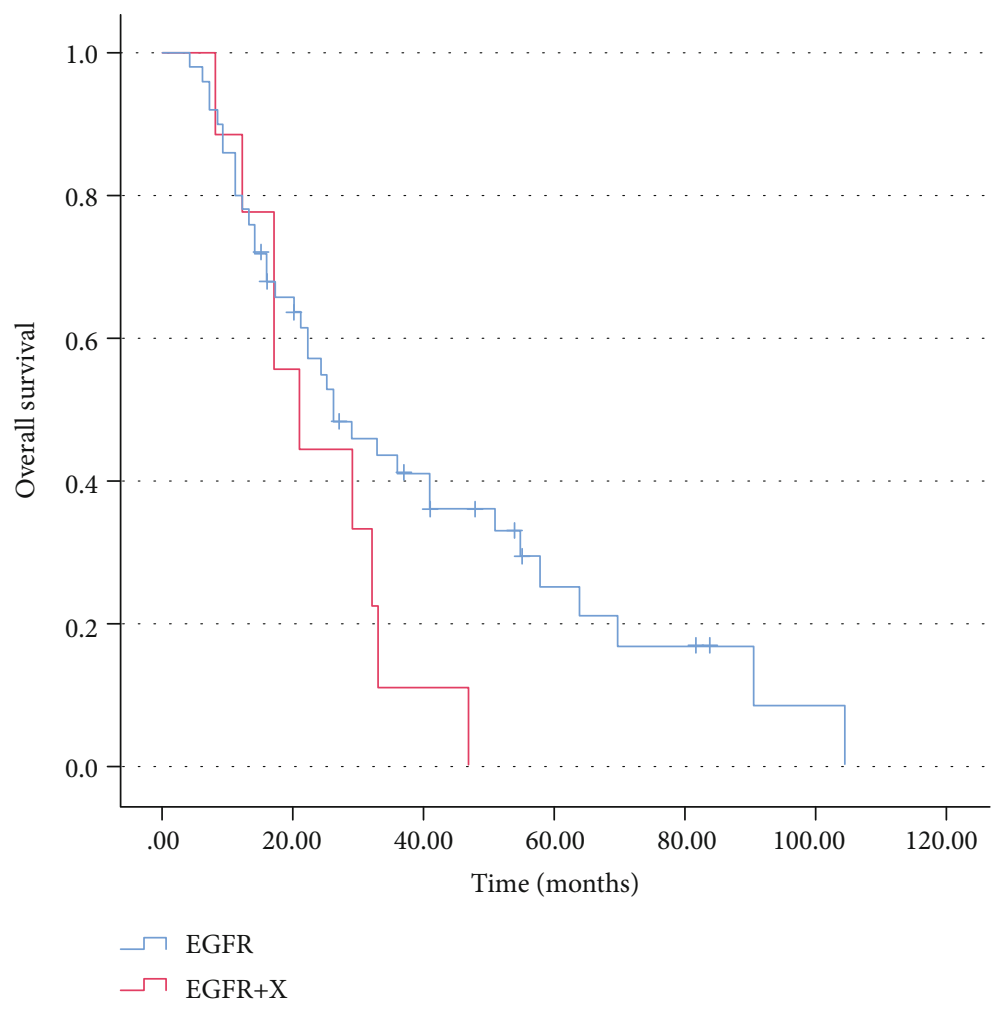

FIGURE 2: Overall survival (OS) in the presence of exclusively EGFR mutation cases compared to coexisting mutations with EGFR patients: OS $=21.0$ vs. 16.0 months, $p=0.104$.

remaining two cases were PIK3CA + KRAS/HER2 mutation (Table 5). EGFR+PIK3CA and EGFR+PTEN were the most common forms of coexistence, accounting for $28.57 \%$ $(4 / 14)$ and $21.43 \%(3 / 14)$, respectively, and we also found 1 case of EGFR+KRAS coexistence. These results indicated that downstream pathways engaged by EGFR can be activated by certain genomic changes.

In patients with EGFR mutation, we found an adverse effect of a concomitant mutation on prognosis. The median OS to TKIs had higher trends in exclusively EGFR mutation cases when compared to coexisting mutations with EGFR tumors ( $p>0.05$ without statistical significance). Of the 9 patients with complete follow-up data, coexisting genes in most patients were PIK3CA and PTEN (5 PIK3CA + EGFR and 3 PTEN+EGFR). PIK3CA encodes PI3Ks of the IA class activated by growth factor receptor tyrosine kinases [33]. Aberrant activation of $\mathrm{PI} 3 \mathrm{~K} / \mathrm{AKT} / \mathrm{mTOR}$ pathway is one of the mechanisms of acquired resistance to EGFR-TK inhibitors in patients with adenocarcinoma carrying EGFR activating mutations [34]. In gefitinib-sensitive lung cancer cells with EGFR mutations and amplifications, continued activation of PI3K signaling by the PIK3CA oncogenic mutant was sufficient to abrogate gefitinib-induced apoptosis [35]. Shorter median survival was reported in patients with concomitant PIK3CA and EGFR mutations, suggesting that the presence of PIK3CA mutations may be a predictor of poor prognosis in patients with EGFR mutations [36]. Guibert et al. found patients with EGFR/PIK3CA mutations experienced worse PFS than did patients with only EGFR mutations [37]. PTEN used to be believed as a classic tumor suppressor, low expression of PTEN protein due to gene mutation or missing may excessively activate PIK3CA/AKT signal pathway and drive the process of development and metastasis of tumor [38], which related to poor prognosis of nonsmall cell lung cancer and TKIs resistance $[39,40]$. For NSCLC patients with EGFR-sensitive mutations, patients with concurrent PTEN deletion mutation had a worse prognosis after TKI treatment than those with complete PTEN [41]. It has been shown that PTEN deletion and low PTEN protein expression were predictors of poor outcome in patients treated with EGFR-TKIS [39]. In our study, two patients with PIK3CA + KRAS/HER2 mutations were lost to follow-up and therefore no prognostic data were available. In a previous small cohort study, there was a shorter median survival in patients with a coexisting mutation (EGFR, KRAS, BRAF, and ALK) versus those with mutations in PIK3CA alone [29].

This is a descriptive study that is aimed at describing the genetic variations of advanced Chinese lung adenocarcinoma patients. Our data show a panoramagram of mutation pattern in Chinese stage IV lung adenocarcinoma patients. $31.58 \%$ of patients harbored multiple mutations in our cohort, which are often related to TKI resistance and poor prognosis, so whole-genome sequencing is an effective method for making appropriate treatment choices. NGS ctDNA analysis could detect genomic mutations in NSCLC patients efficiently, especially when tumor progression occurs and positive treatment adjustments need to be made. Although not all patients received 425 gene panel detection, several unknown mutations were identified which were 
potential targets for TKI therapy. We detected extensive sequencing in the real-world cohort, but the limitation was the small sample size, and further large sample studies are needed to confirm these findings.

\section{Data Availability}

The [population characteristics and gene sequencing] data used to support the findings of this study are included within the article.

\section{Conflicts of Interest}

The authors declare that they have no conflicts of interest.

\section{References}

[1] F. Bray, J. Ferlay, I. Soerjomataram, R. L. Siegel, L. A. Torre, and A. Jemal, "Global cancer statistics 2018: GLOBOCAN estimates of incidence and mortality worldwide for 36 cancers in 185 countries," CA: a Cancer Journal for Clinicians, vol. 68, no. 6, pp. 394-424, 2018.

[2] N. Howlader, G. Forjaz, M. J. Mooradian et al., "The effect of advances in lung-cancer treatment on population mortality," The New England Journal of Medicine, vol. 383, no. 7, pp. 640-649, 2020.

[3] B. J. Solomon, T. Mok, D. W. Kim et al., "First-line crizotinib versus chemotherapy in ALK-positive lung cancer," The New England Journal of Medicine, vol. 371, no. 23, pp. 2167-2177, 2014.

[4] J. C.-H. Yang, Y.-L. Wu, M. Schuler et al., "Afatinib versus cisplatin-based chemotherapy for_EGFR_mutation-positive lung adenocarcinoma (LUX-Lung 3 and LUX-Lung 6): analysis of overall survival data from two randomised, phase 3 trials," The Lancet Oncology, vol. 16, no. 2, pp. 141-151, 2015.

[5] D. S. Ettinger, D. E. Wood, C. Aggarwal et al., "NCCN guidelines insights: non-small cell lung cancer, version 1.2020," Journal of the National Comprehensive Cancer Network, vol. 17, no. 12, pp. 1464-1472, 2019.

[6] C. Aggarwal, J. C. Thompson, T. A. Black et al., "Clinical implications of plasma-based genotyping with the delivery of personalized therapy in metastatic non-small cell lung cancer," JAMA Oncology, vol. 5, no. 2, pp. 173-180, 2019.

[7] S. Torquato, A. Pallavajjala, and A. Goldstein, "Genetic mutations detected in cell-free DNA are associated with enzalutamide and abiraterone resistance in castration-resistant prostate cancer," JCO Precision Oncology, vol. 3, 2019.

[8] A. M. Bolger, M. Lohse, and B. Usadel, "Trimmomatic: a flexible trimmer for Illumina sequence data," Bioinformatics, vol. 30, no. 15, pp. 2114-2120, 2014.

[9] D. C. Koboldt, Q. Zhang, D. E. Larson et al., "VarScan 2: somatic mutation and copy number alteration discovery in cancer by exome sequencing," Genome Research, vol. 22, no. 3, pp. 568-576, 2012.

[10] K. C. Amarasinghe, J. Li, S. M. Hunter et al., "Inferring copy number and genotype in tumour exome data," Genomics, vol. 15 , no. 1, p. $732,2014$.

[11] M. Takeda, K. Sakai, M. Terashima et al., "Clinical application of amplicon-based next-generation sequencing to therapeutic decision making in lung cancer," Annals of Oncology, vol. 26, no. 12, pp. 2477-2482, 2015.
[12] W. W. J. de Leng, C. G. Gadellaa-van Hooijdonk, F. A. S. Barendregt-Smouter et al., "Targeted next generation sequencing as a reliable diagnostic assay for the detection of somatic mutations in tumours using minimal DNA amounts from formalin fixed paraffin embedded material," PLoS One, vol. 11, no. 2, article e0149405, 2016.

[13] R. Kamps, R. Brandão, B. Bosch et al., "Next-generation sequencing in oncology: genetic diagnosis, risk prediction and cancer classification," International Journal of Molecular Sciences, vol. 18, no. 2, p. 308, 2017.

[14] A. B. Schrock, A. Welsh, J. H. Chung et al., "Hybrid capturebased genomic profiling of circulating tumor DNA from patients with advanced non-small cell lung cancer," Journal of Thoracic Oncology, vol. 14, no. 2, pp. 255-264, 2019.

[15] M. C. Schwaederlé, S. P. Patel, H. Husain et al., "Utility of genomic assessment of blood-derived circulating tumor DNA (ctDNA) in patients with advanced lung adenocarcinoma," Clinical Cancer Research, vol. 23, no. 17, pp. 51015111, 2017.

[16] R. Rosell, T. Moran, C. Queralt et al., "Screening for epidermal growth factor receptor mutations in lung cancer," The New England Journal of Medicine, vol. 361, no. 10, pp. 958-967, 2009.

[17] S. P. D'Angelo, M. C. Pietanza, M. L. Johnson et al., "Incidence of EGFR exon 19 deletions and L858R in tumor specimens from men and cigarette smokers with lung adenocarcinomas," Journal of Clinical Oncology, vol. 29, no. 15, pp. 2066-2070, 2011.

[18] S. Li, L. Li, Y. Zhu et al., "Coexistence of EGFR with KRAS, or BRAF, or PIK3CA somatic mutations in lung cancer: a comprehensive mutation profiling from 5125 Chinese cohorts," British Journal of Cancer, vol. 110, no. 11, pp. 2812-2820, 2014.

[19] S. Watanabe, Y. Minegishi, H. Yoshizawa et al., "Effectiveness of gefitinib against non-small-cell lung cancer with the uncommon EGFR mutations G719X and L861Q," Journal of Thoracic Oncology, vol. 9, no. 2, pp. 189-194, 2014.

[20] C. H. Chiu, C. T. Yang, J. Y. Shih et al., "Epidermal growth factor receptor tyrosine kinase inhibitor treatment response in advanced lung adenocarcinomas with G719X/L861Q/S768I mutations," Journal of Thoracic Oncology, vol. 10, no. 5, pp. 793-799, 2015.

[21] J. C.-H. Yang, L. V. Sequist, S. L. Geater et al., "Clinical activity of afatinib in patients with advanced non-small-cell lung cancer harbouring uncommon _EGFR_mutations: a combined posthoc analysis of LUX-Lung 2, LUX-Lung 3, and LUX-Lung 6," The Lancet Oncology, vol. 16, no. 7, pp. 830-838, 2015.

[22] E. Banno, Y. Togashi, Y. Nakamura et al., "Sensitivities to various epidermal growth factor receptor-tyrosine kinase inhibitors of uncommon epidermal growth factor receptor mutations L861Q and S768I: what is the optimal epidermal growth factor receptor-tyrosine kinase inhibitor," Cancer Science, vol. 107, no. 8, pp. 1134-1140, 2016.

[23] C. H. Yun, K. E. Mengwasser, A. V. Toms et al., "The T790M mutation in EGFR kinase causes drug resistance by increasing the affinity for ATP," Proceedings of the National Academy of Sciences of the United States of America, vol. 105, no. 6, pp. 2070-2075, 2008.

[24] M. Saito, K. Shiraishi, H. Kunitoh, S. Takenoshita, J. Yokota, and T. Kohno, "Gene aberrations for precision medicine against lung adenocarcinoma," Cancer Science, vol. 107, no. 6, pp. 713-720, 2016. 
[25] G. J. Riely, M. G. Kris, D. Rosenbaum et al., "Frequency and distinctive spectrum of KRAS mutations in never smokers with lung adenocarcinoma," Clinical Cancer Research, vol. 14, no. 18, pp. 5731-5734, 2008.

[26] Y. Zhao, S. Wang, B. Zhang et al., "Clinical management of non-small cell lung cancer with concomitant EGFR mutations and ALK rearrangements: efficacy of EGFR tyrosine kinase inhibitors and crizotinib," Targeted Oncology, vol. 14, no. 2, pp. 169-178, 2019.

[27] L. La Fleur, E. Falk-Sörqvist, P. Smeds et al., "Mutation patterns in a population-based non-small cell lung cancer cohort and prognostic impact of concomitant mutations in _KRAS_ and _TP53_or_STK11_,"Lung Cancer, vol. 130, pp. 50-58, 2019.

[28] J. M. Spoerke, C. O'Brien, L. Huw et al., "Phosphoinositide 3kinase (PI3K) pathway alterations are associated with histologic subtypes and are predictive of sensitivity to PI3K inhibitors in lung cancer preclinical models," Clinical Cancer Research, vol. 18, no. 24, pp. 6771-6783, 2012.

[29] J. E. Chaft, M. E. Arcila, P. K. Paik et al., "Coexistence of PIK3CA and other oncogene mutations in lung adenocarcinoma-rationale for comprehensive mutation profiling," Molecular Cancer Therapeutics, vol. 11, no. 2, pp. 485491, 2012.

[30] A. Marchetti, C. Martella, L. Felicioni et al., "EGFR mutations in non-small-cell lung cancer: analysis of a large series of cases and development of a rapid and sensitive method for diagnostic screening with potential implications on pharmacologic treatment," Journal of Clinical Oncology, vol. 23, no. 4, pp. 857-865, 2005.

[31] G. Alì, A. Proietti, S. Pelliccioni et al., "ALK rearrangement in a large series of consecutive non-small cell lung cancers: comparison between a new immunohistochemical approach and fluorescence in situ hybridization for the screening of patients eligible for crizotinib treatment," Archives of Pathology \& Laboratory Medicine, vol. 138, no. 11, pp. 1449-1458, 2014.

[32] L. M. Sholl, D. L. Aisner, M. Varella-Garcia et al., "Multi-institutional oncogenic driver mutation analysis in lung adenocarcinoma: the lung cancer mutation consortium experience," Journal of Thoracic Oncology, vol. 10, no. 5, pp. 768-777, 2015.

[33] A. C. Tan, "Targeting the PI3K/Akt/mTOR pathway in nonsmall cell lung cancer (NSCLC)," Thoracic Cancer, vol. 11, no. 3, pp. 511-518, 2020.

[34] C. Fumarola, M. A. Bonelli, P. G. Petronini, and R. R. Alfieri, "Targeting PI3K/AKT/mTOR pathway in non small cell lung cancer," Biochemical Pharmacology, vol. 90, no. 3, pp. 197207, 2014.

[35] J. A. Engelman, T. Mukohara, K. Zejnullahu et al., "Allelic dilution obscures detection of a biologically significant resistance mutation in EGFR-amplified lung cancer," The Journal of clinical investigation, vol. 116, no. 10, pp. 2695-2706, 2006.

[36] M. Tiseo, M. Bersanelli, F. Perrone et al., "Different clinical effects upon separate inhibition of coexisting EGFR and PI3KCA mutations in a lung adenocarcinoma patient," Lung Cancer, vol. 87, no. 2, pp. 204-206, 2015.

[37] N. Guibert, F. Barlesi, R. Descourt et al., "Characteristics and outcomes of patients with lung cancer harboring multiple molecular alterations: results from the IFCT study biomarkers France," Journal of Thoracic Oncology, vol. 12, no. 6, pp. 963973, 2017.

[38] T. Maehama and J. E. Dixon, "The tumor suppressor, PTEN/MMAC1, dephosphorylates the lipid second messen- ger, phosphatidylinositol 3,4,5-trisphosphate," The Journal of Biological Chemistry, vol. 273, no. 22, pp. 13375-13378, 1998.

[39] G. Bepler, S. Sharma, A. Cantor et al., "RRM1 and PTEN as prognostic parameters for overall and disease-free survival in patients with non-small-cell lung cancer," Journal of Clinical Oncology, vol. 22, no. 10, pp. 1878-1885, 2004.

[40] Y. Kokubo, A. Gemma, R. Noro et al., "Reduction of PTEN protein and loss of epidermal growth factor receptor gene mutation in lung cancer with natural resistance to gefitinib (IRESSA)," British Journal of Cancer, vol. 92, no. 9, pp. 1711-1719, 2005.

[41] F. Wang, X.-Y. Diao, X. Zhang et al., "Identification of genetic alterations associated with primary resistance to EGFR-TKIs in advanced non-small-cell lung cancer patients with EGFR sensitive mutations," Cancer Communications, vol. 39, no. 1, p. 7, 2019. 